\section{Imaging sketch of bedside ultrasonography to improve the diagnosis of rupture of splenic artery aneurysm}

\author{
Bahjat Barakat, ${ }^{1}$ Raffaele Pezzilli ${ }^{2}$ \\ 'Department of Emergency, S. Orsola- \\ Malpighi University Hospital, Bologna; \\ 2Department of Digestive System, S. \\ Orsola-Malpighi University Hospital, \\ Bologna, Italy
}

Splenic artery aneurysm (SAA) is a rare cause of upper abdominal pain. In autopsy studies, its incidence ranges from 0.01 to $0.2 \%$ and increases to $10 \%$ in older patients, ${ }^{1}$ also, the incidence is higher in female gender as compared to male gender with a ratio of $4: 1{ }^{2}$ The diagnosis of this disease is clinically difficult without imaging. ${ }^{3-6}$ Due to rarity of this disease, we considered noteworthy to report the imaging, which lies to a diagnosis of SAA in a 58-year-old woman who was admitted to Emergency Room for the rapid onset of epigastric pain, not associated with other symptoms. She was previously cholecystectomized for gallstones and she did not take any medication. The clinical parameters were within the normal limits: arterial blood pressure was $110 / 60 \mathrm{mmHg}$, blood oxygen saturation levels (99\%), cardiac rate 60 b.p.m., and body temperature of $36^{\circ} \mathrm{C}$. The electrocardiogram was normal as well as the cardiac and pulmonary physical examination. There was a mild tenderness of the abdomen without signs of peritoneal irritation or pulsatile mass. There was pallor of the skin. The biochemistry revealed an increase of white cell count $(13.990 \mathrm{mmc})$, a reduced levels of $\mathrm{Hb}(10.9 \mathrm{~g} / \mathrm{dL})$ and hematocrit (32.8\%). Liver and renal parameters were within the normal limits as well as the coagulation. Both the chest and the abdomen x-rays were normal. The abdominal ultrasound showed no alterations of the liver, biliary tree, kidneys, pancreas (head and body), whereas there was the presence of a small anechoic area near to the tail of the pancreas (Figure 1); in addition, hypoechoic spots of the spleen and a moderate abdominal fluid collection were identified (Figure 2). An ultrasound-color Doppler and pulse-Doppler of the abdominal vessels were carried out showing an aneurysmatic dilation of the splenic artery (Figure 3), and a turbulent flow within the splenic artery aneurysm (Figure 4). The patient was rehydrated with fluids and a computed tomography was carried out. The contrast-enhanced computed tomography showed multiple infarctions of the spleen and bloody abdominal fluid collection and an aneurysm of $2 \mathrm{~cm}$ of diameter of the SAA (Figures 5 and 6 ). The patient became critically in the subsequent 2 hours after admission and she underwent laparotomy. At the surgical exploration, blood was found in both the peritoneal cavity and in lesser sac as well as a rupture of the splenic artery was identified. Thus, a ligation of the proximal portion of the artery together with resection of the aneurysm and spleen was carried out. The pathological examination of the resected specimen showed a perforated aneurysm of the splenic artery and multiple small splenic infarction areas.

Splenic artery aneurysm is a rare and potentially life-treating disease ${ }^{7}$ and its diagnosis should be kept in mind in all those patients
Correspondence: Raffaele Pezzilli, Department of Digestive System, Sant'Orsola-Malpighi Hospital, via Massarenti 9, 40138 Bologna, Italy.

Tel: +39.051.636.4148 - Fax: +39.051.636.4148.

E-mail: raffaele.pezzilli@aosp.bo.it

Key words: Splenic aneurysm; Ultrasonography; Ultrasound-color Doppler; Ultrasound pulseDoppler; Computer tomography.

Conflict of interest: the authors declare no potential conflict of interest.

Received for publication: 27 April 2015 Accepted for publication: 20 May 2015.

This work is licensed under a Creative Commons Attribution 3.0 License (by-nc 3.0).

(C) Copyright B. Barakat and R. Pezzilli, 2015 Licensee PAGEPress, Italy

Emergency Care Journal 2015; 11:5257

doi:10.4081/ecj.2015.5257

having a sudden onset pain in the upper abdomen with of without arterial hypotension. The risk factors of SAA include portal hypertension, congenital abnormalities of the vessels, vascular and connective vascular disorders, vascular trauma, or degenerative arterial diseases; ${ }^{8}$ it has been also found in pregnant women and in this latter case the maternal and fetal mortality rates are as high as $70-90 \%$, respectively. ${ }^{9-11}$ However, in our case none of these factors were clinically apparent. Emergency surgical management may improve survival of these kinds of patients. ${ }^{5}$ An alternative approach to SAA is endovascular treatment that should be preferred for those patients

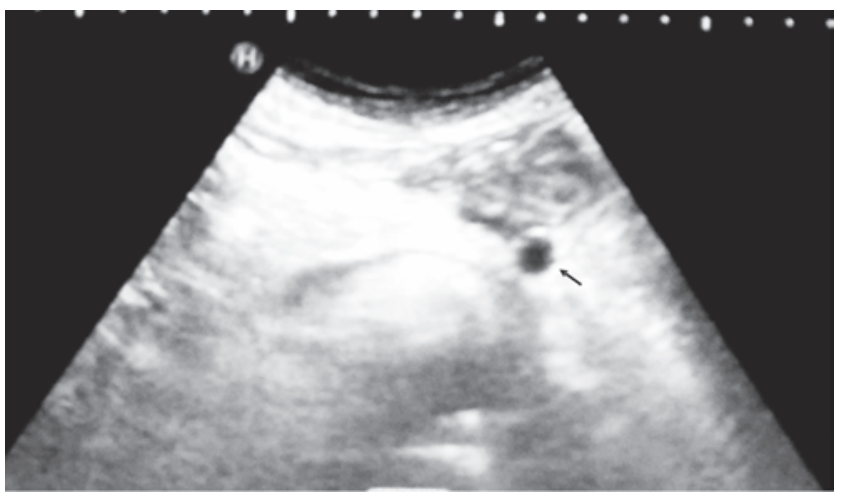

Figure 1. Ultrasonography: small anechoic area near to the tail of the pancreas compatible with aneurysm of the splenic artery (arrow).

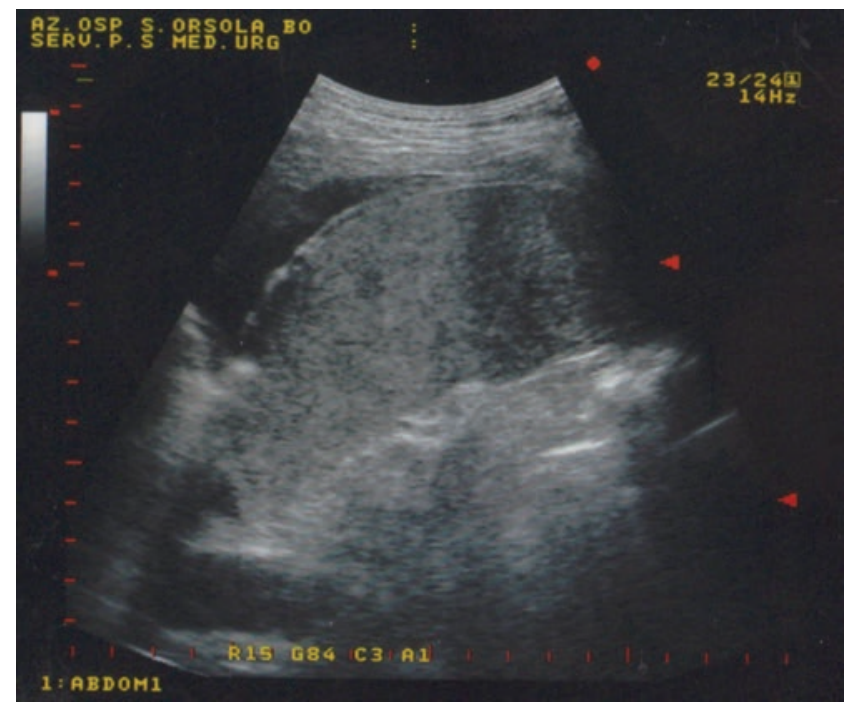

Figure 2. Ultrasonography: hypoechoic spots of the spleen and a moderate abdominal fluid collection. 


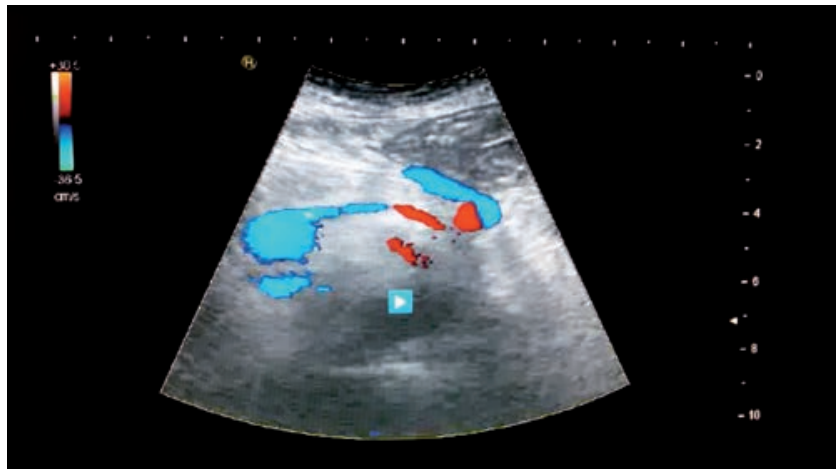

Figure 3. Ultrasound-color Doppler showing an aneurysmatic dilation of the splenic artery.

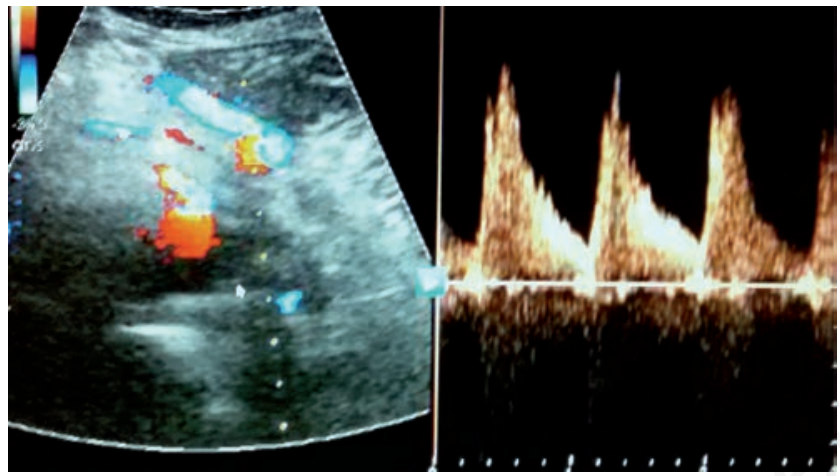

Figure 4. Ultrasound pulse-Doppler of the abdominal vessels showing a turbulent flow within the splenic artery aneurysm.

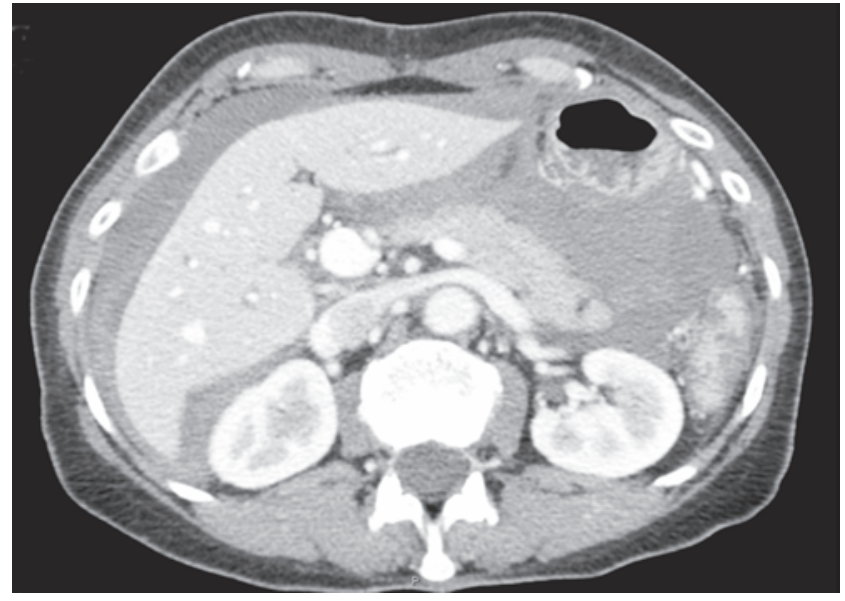

Figure 5. Contrast-enhanced computed tomography showing multiple infarctions of the spleen and bloody abdominal fluid collection.

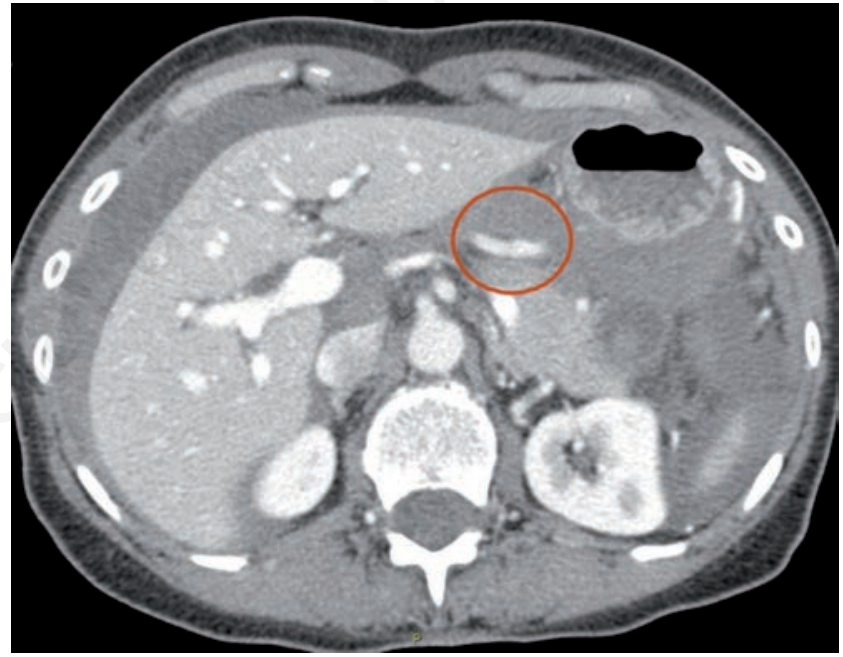

Figure 6. Contrast-enhanced computed tomography showing an aneurysm of $2 \mathrm{~cm}$ of diameter of the splenic artery aneurism (red circle). unfit for surgery or those who may be treated in elective settings. ${ }^{5,9}$ Nevertheless, a correct diagnosis, especially using bedside ultrasonography, significantly improves the correct management of patients having SAA in Emergency Room.

\section{References}

1. Reardon PR, Otah E, Craig ES, et al. Laparoscopic resection of splenic artery aneurysms. Surg Endosc 2005;19:488-93.

2. Dave SP, Reis ED, Hossain A, et al. Splenic artery aneurysm in the $1990 \mathrm{~s}$. Ann Vasc Surg 2000;14:223-9.
3. Betal D, Khangura JS, Swan PJ, Mehmet V. Spontaneous ruptured splenic artery aneurysm: a case report. Cases J 2009;2: 7150.

4. Sarikaya S, Ekci B, Aktas C, et al. A rare clinic presentation of abdominal pain: rupture of splenic artery aneurysm: a case report. Cases J 2009;2:148.

5. Abdulrahman A, Shabkah A, Hassanain M, Aljiffry M. Ruptured spontaneous splenic artery aneurysm: a case report and review of the literature. Int $\mathrm{J}$ Surg Case Rep 2014;5:754-7.

6. Deshpande AA, Kulkarni VM, Rege S, et al. Ruptured true aneurysm of the splenic artery: an unusual cause of haemoperitoneum. J Postgrad Med 2000;46:191-2.

7. Mattick A, Gawthrope I. Splenic artery aneurysm rupture: case report of this uncommon presentation. BMJ Case Rep 2009;2009:bcr10.2008.1148.

8. Selo-Ojeme D0, Welch CC. Review: spontaneous rupture of splenic artery aneurysm in pregnancy. Eur $\mathrm{J}$ Obstet Gynecol Reprod Biol 2003;109:124-7.

9. Carey K, Northcutt A, Bhullar I. Successful management of delayed splenic rupture with angioembolization. Am Surg 2014;80: e265-7.

10. Khurana J, Spinello IM. Splenic artery aneurysm rupture: a rare but fatal cause for peripartum collapse. J Intensive Care Med 2013;28:131-3.

11. Sadat U, Dar 0, Walsh S, Varty K. Splenic artery aneurysms in pregnancy. A systematic review. Int J Surg 2008;6:261-5. 